\title{
The Bidirectional Effects of Obsessive-Compulsive Symptoms and Difficulties in Emotion Regulation in Chinese Adults During the COVID-19 Pandemic: A Dynamic Structural Equation Model
}

\author{
Danping Hong \\ Sun Yat-sen University \\ Yawen Zhu \\ Northwest Normal University \\ Runting Chen \\ Sun Yat-sen University \\ Bihong Xiao \\ Sun Yat-sen University \\ Yueyi Huang \\ Sun Yat-sen University \\ Meng Yu ( $\square$ yumeng23@mail.sysu.edu.cn ) \\ Sun Yat-sen University
}

Research Article

Keywords: COVID-19, Difficulties in Emotion Regulation, Dynamic Structural Equation Modeling, Obsessive-Compulsive Symptoms, Sleep

Problems

Posted Date: September 29th, 2021

DOI: https://doi.org/10.21203/rs.3.rs-886323/v1

License: (c) (7) This work is licensed under a Creative Commons Attribution 4.0 International License. Read Full License 


\section{Abstract}

Background: With the accumulation of negative emotions bought by COVID-19-related dysfunctional beliefs, individuals adopted obsessivecompulsive (OC) symptoms (e.g., over-checking the wearing of masks) and formed difficulties in emotion regulation (DER). Both OC symptoms and DER had a devastating impact on the individual's mental health. This study focused on the temporal dynamics of the bidirectional relation between $\mathrm{OC}$ symptoms and DER. As an extension, we further explored whether OC and DER and their relationship affect sleep problems (SP).

Methods: In February 2020, a 14-day (twice a day) online questionnaire survey was conducted on 122 Chinese adults (aged 18 to 55 years; 63 females). In addition, this research applied a dynamic structural equation model with a cross-lagged relationship and a time series. Health anxiety, anxiety, and depression were controlled as covariates.

Results: Both OC symptoms and DER had a significant autoregressive and cross-lagged effect. Comparatively speaking, DER was a stronger predictor of OC symptom than OC's prediction of DER. Besides, both higher levels of OC symptoms and DER were related to the severity of SP.

Conclusions: More guidance on identifying intervening $\mathrm{OC}$ symptoms and emotion regulation should be added to reduce the external crisis's negative impact on public mental health.

\section{Introduction}

At the end of January 2020, with the massive outbreak of COVID-19 in China, the government called the populace at home and not necessarily go outside. Getting in and out of the streets, public transport, and other places, the public needed to wear masks, detect body temperature, and maintain social distance, which became the moment's social behavior norms to counter the threat from virus transmission.

Obsessive-Compulsive (OC) symptoms are composed of intrusive thoughts and repetitive behaviors, essentially trying to suppress or prevent excessive emotional expression (Foa et al., 1998; American Psychiatry Association, 2013). OC symptoms incorporating COVID-19-related social norms manifested as over-checking, over-washing, obsessing, and metal neutralizing. Several kinds of dysfunctional beliefs might induce negative emotions and evaluations, such as, exaggerating external threats, paying too much attention to the correctness of their thoughts and trying to control them, not tolerating uncertainty and perfectionism. Combined with the context of the epidemic, when intrusive thoughts (e.g., exaggerating the possibility of infection with a virus) came, compulsive behaviors would be used to alleviate the pain of negative emotions temporarily. However, the repeated execution of the compulsions can also accumulate painful emotions in the long run, especially when people lack understanding of emotions and confidence in regulating painful emotions (Luterek, Mennin, \& Fresco, 2005; Calkins et al., 2013). Therefore, due to these negative beliefs, people would continue to use compulsive behaviors, resulting in a vicious circle. Repeated OC symptoms will solidify into OC disorders (Stern et al., 2014).

Difficulties in Emotion Regulation (DER) measures whether an individual responds to negative emotions in an unbalanced way (Gratz \& Roemer, 2004). Gratz and Roemer (2004) identified four aspects involved in emotion dysregulation: (1) poor awareness and understanding of emotions, (2) poor acceptance of emotions, (3) lack of the ability to engage in goal-directed behavior, and refrain from impulsive behavior, when experiencing negative emotions, and (4) access to maladaptive emotion regulation strategies. As indicating by previous studies, avoiding internal experience is the basis of many psychological symptoms, and DER is directly related to various psychopathological symptoms (Hu et al., 2014). In the face of crises and a forced social quarantine, the public needs to adjust their negative emotions promptly. It is speculated that individuals with DER are more difficult in adapting to the epidemic environment and quickly suffer from psychological problems (e.g., Groarke et al., 2021).

Dominated by cross-sectional studies, most existing findings have confirmed the intimate relationship between OC and DER. Gross (1998) proposed that the process of emotion regulation is shown as "situation selection $\rightarrow$ allocation of attention $\rightarrow$ appraisal $\rightarrow$ response." Calkins et al. (2013) expanded this process model and considers that OC symptoms, especially compulsions, are a maladaptive emotion regulation strategy. It takes suppression during the response process, in which people try to reduce emotional expression. Furthermore, Moritz et al. (2018) pointed out that the recurrence of OC symptoms may be due to a lack of adaptive coping rather than an excess of maladaptive coping. Stern et al. (2014) conducted a study on undergraduates $(n=170)$ and results showed that OC symptoms were significantly correlated with poor understanding and fear for both negative and positive emotions.

In addition, DER would promote and maintain OC symptoms. For instance, in clinical samples $(n=59)$ and non-clinical samples $(n=331)$, even if anxiety, depression, and demographic variables were controlled, the positive correlation between OC and DER was still established (Yap et al., 2018). In another non-clinical sample, Fergus and Bardeen (2014) showed that difficulties in impulse control and lack of 
emotional clarity were uniquely associated with each dimension of OC symptoms. Yap et al. (2018) suggested that non-acceptance of emotions and non-participation in goal-oriented behaviors were markedly associated with OC across samples.

So far, however, no longitudinal finding has pointed out the bidirectional relationship between OC symptoms and DER. Although some crosssectional studies obtained a significant mediation path that DER affected OC, this inference was not causally persuasive. For instance, Eichholz et al. (2020) confirmed that DER played a mediating role in self-compassion affecting OC symptom severity in patients $(n=90)$. Combining the previous research and the epidemic's situation, it can be inferred that they are more likely to present a mutual influence. When individuals lack effective and adaptive emotion regulation strategies during the epidemic, they would rely on current social norms to alleviate negative emotions. Influenced by dysfunctional beliefs, intrusive thoughts would make individuals be more inclined to adopt maladaptive compulsions to avoid, un-clarify, uncomprehend and un-accept their negative and distressed emotions, which further promote the formation of DER. Besides, when this vicious circle begins, it makes them be less confident in regulating emotions and difficult to control their impulsive behaviors as well. To conclude, discussing the relationship between OC and DER under the epidemic environment would help guide the public in informing appropriate response strategies when facing an emergency public crisis.

Following the predecessors' suggestion, when discussing the relationship between $\mathrm{OC}$ and DER, the effects of anxiety and depression should be considered covariates. And anxiety and depression were reported to be closely related to both (e.g., Yap et al., 2018). When confronted with the health threats posed by COVID-19, individuals are prone to worry about the health of themselves and their close persons, which quickly leads to health anxiety (Asmundson \& Taylor, 2020). Health anxiety (HA) refers to the state that people overly worry about getting sick, exaggerate their physical feelings, and negatively explain physical symptoms (Salkovskis \& Warwick, 1986). According to the cognitive model of HA (Salkovskis \& Warwick, 1986), it will further produce distorted beliefs, destructive emotions, and maladaptive behaviors, resulting in more OC symptoms and DER (e.g., Sunderland et al., 2013; Görgen et al., 2014). During the COVID-19 lockdown, individuals barely went to the hospital for a diagnosis in time or relied on work to be distracted. In that case, they were supposed to be more likely to use compulsions to get rid of emotional distress. Therefore, HA may also play an essential role in the relationship between the association of OC symptoms and DER.

Sleep problems are also an important topic that cannot be ignored during social interaction (Altena et al., 2020). As an extension, we explored the predictive effect of the relationship between the OC symptoms and DER on daily sleep. Sleep problems (SP), including difficulty in falling asleep, waking up early, and not getting enough sleep were the COVID-19-related features, which are also shown to be part of the characteristics of sleep disorders in DSM-5 (American Psychiatric Association, 2013). Past research has found intrusive thoughts, uncontrollable worries, and other cognitive arousals that may hinder sleep onset and then cause insomnia (Harvey, 2000). Riemann et al. (2010) proposed a hyperarousal model of insomnia. It was pointing out that psychological stress before going to bed (e.g., intrusive thoughts) and the dysregulation of emotion regulation are accompanied by excessive reflection, which leads to a variety of SP. In a crosssectional study with non-clinical samples, similar results were confirmed, especially, that obsessions aggravated insomnia (Timpano et al., 2014). And compulsions are also a good predictor of sleep time reduction and sleep loss (Alfano \& Kim, 2011). Moreover, SP was considered to be related to the accumulation of negative emotions, decreased positive emotions, and insufficient emotional regulation (Tsypes, Aldao, \& Mennin, 2014). A longitudinal study of three years $(n=942)$ has examined that DER positively predicted SP, and DER played an intermediary role in social relationships and SP (Tavernier \& Willoughby, 2015). Moreover, a review of 44 cross-sectional epidemic articles worldwide summarized multiple factors that could lead to SP (Jahrami et al., 2021), such as negative emotions, stress, and the deficiency of social support. Nevertheless, no research has discussed OC symptoms in cross-sectional studies and used longitudinal analysis during the COVID-19 pandemic. It can be inferred that both OC and DER could affect SP, and even OC would further impact SP through DER.

Until now, only a minimal number of studies have combined longitudinal research design to measure the same batch of subjects to psychology changes of OC symptoms or DER. For example, a study in the UK $(n=1958)$ used four-time points to indicate the association between loneliness and depressive symptoms, finding that DER was not the moderator of temporal interaction (Groarke et al., 2021). However, these conventional longitudinal studies collected developmental process data to concern between-person change and covariates affecting change. Ambulatory assessment (AA) highlights its advantage in timely and acutely recording the subtle changes of the public's psychological states (Trull \& Ebner-Priemer, 2013). The stable process data measured by AA would fluctuate around the mean, focusing on within-person variability and covariates predicting when values will deviate from the mean (McNeish \& Hamaker, 2020). It provides daily measurement to assist to draw a more stable causal inference for OC and DER.

To conclude, the present study used an ambulatory assessment design with a sample of 122 Chinese adults to conduct questionnaires twice in the morning and evening for 14 consecutive days, with 28 measurement intervals. The Dynamic Structural Equation Modeling (DSEM; Asparouhov, Hamaker, \& Muthén, 2018) framework was utilized to create a multilevel cross-lagged model that examined the bidirectional relationships between OC symptoms and DER. Next, to ensure the reliability of causal inferences, we also added factors related

Page $3 / 13$ 
to the two variables and the epidemic - the level of health anxiety, anxiety, and depression - as covariates. As a corresponding extension, we tested the predictive effect of the two-way relationship on SP.

\section{Method}

\subsection{Participants}

In total, 122 subjects were included in the final sample. Their age ranged from 18 to $55(M=22.18, S D=7.03)$ with 69 females (56.6\%). Most of them (83.6\%) have a bachelor's degree or above. The sample reported to come from 18 provinces in China, with a total of 50 cities. There were 12 participants (9.8\%) coming from severe epidemic areas and 25 (20.5\%) from extremely severe epidemic areas (See Supplementary Table 1 for more information).

\subsection{Procedures}

The present study was approved by the corresponding author's affiliation ethic review board. The Wenjuanxing platform (https://www.wjx.cn/) was used to issue questionnaires. First, we collected baseline data on mental health variables from the Chinese public from February 1, 2020. Participants volunteered to join our online WeChat group for follow-up research. Secondly, after entering, they were assigned with an experiment number and needed to remember the number and then logged into the questionnaire with the same number each time. Beginning on February 2, at 9 am and 9 pm for 14 consecutive days, the research assistant sent out a questionnaire link to the WeChat group. Participants were asked to complete the questionnaire as soon as possible, which took about 2-3 minutes each time.

\subsection{Measures}

\subsubsection{Daily measures}

Obsessive-Compulsive Symptoms. The study chose the four items that fitted the pandemic situation from Obsessive-Compulsive InventoryRevised (OCl-R; Foa et al., 2002; Tang et al., 2011), including washing, checking, obsessing, and mental neutralizing. The scale is a five-point score from 1 (not at all) to 5 (extremely). In the present study, Intraclass Correlation (ICC; Muthen, 2019) Coefficient was adopted to represent the within-person differences accounted for the proportion of the total variance, which was often used to assess the fit of stratified data in ambulatory assessment research. The ICC for OC symptoms was .90.

Difficulties in Emotion Regulation. The Chinese version of the Difficulties in Emotion Regulation Scale (DERS; Gratz \& Roemer, 2004; Li et al., 2018) was used and was selected one item in each of its six dimensions. The adapted version used in the presents study included difficulties in engaging directed behavior, limited access to emotional regulation strategies, nonacceptance of emotional responses, difficulties in controlling impulsive behaviors under negative emotions, lack of emotional clarify, and lack of emotional awareness. We adapted the pandemic's context and changed it to "When I feel pressure due to the epidemic." It is a 5-point Likert scale, with a score ranging from 1 (totally disagree) to 5 (totally agree). The ICC for DER was .85.

Sleep problems. The assessment combined the Insomnia Severity Index (ISI; Morin, 1993) and the PROMIS Sleep Disturbance-Short Form (Yu et al., 2011). Three questions were selected: (1) difficulties with falling asleep; (2) waking up too early; (3) difficulties with staying asleep. The score ranged from 1 (completely inconsistent) to 5 (very consistent), and it was only measured once a day in the morning. The ICC for SP was .75. The higher the score of the item, the more serious the sleep problems. According to the suggestion of Asparouhov et al. (2018), SP's value was proportionally processed as a categorical variable in the between-level. Specifically, the severity of daily sleep problems accounting for 14 days was divided into mild, moderate, and severe.

\subsubsection{Baseline measures}

Health anxiety. The Chinese version of the Short Health Anxiety Inventory contains 18 items with good reliability and validity (SHAl; Salkovskis, Rimes, \& Warwick, 2002; Zhang et al., 2015). And it consists of 4 statements ranging from 0 (/ spend very little time) to 3 (/ spend most of my time). The internal consistent coefficient was .88 .

Anxiety. The Self-Rating Anxiety Scale (SAS) is widely used and has good reliability and validity (Zung, 1971; Duan \& Sheng, 2012). It contains 20 questions, from 1 (/ spend very little time) to 4 (/ spend most of my time) to score. The Cronbach's alpha in the current study was .73.

Depression. The PROMIS Emotional Distress-Depression-Short Form was used to measure participants' depressive symptoms (Amtmann et al., 2014). The CFA model fitting indexes of this scale can be verified in another adult sample $(n=1,733)$ collected by the team during the 
same period, i.e., $x^{2} / d f=4.782, \mathrm{CFI}=.995, \mathrm{TLI}=.991$, RMSEA $=.037, \mathrm{SRMR}=.011$, which showed a well-fit model (Hoyle, 2012). The scale contains eight questions, scoring from 1 (never) to 5 (almost always). The Cronbach's alpha of the present study was .81.

\subsection{Data analyses}

First of all, IBM SPSS Version 25 conducted descriptive and correlational analyses. Then, we built a DSEM using Mplus 8.3 in the present study. Based on the Bayes estimator, DSEM is a simulation method with posterior distribution, combining the advantages of structural equation modeling, multilevel modeling, autoregressive cross-lagged modeling, and time-series modeling (Asparouhov et al., 2018). DSEM is an effective statistical method for analyzing intensive longitudinal data (ILD; Hamaker et al., 2018), which allows the operating bivariate models simultaneously time. It has good applicability for small sample models (Schuurman et al., 2016). Specifically, 50,000 Markov Chain Monte Carlo (MCMC) iterations and two chains were used to estimate parameters and handle missing data (Gelman et al., 2013). To be specific, the current study contained a total of 122 samples $(M)$ and 28-time observation points ( $T$, every 12-hour), which was considered to have good statistical reliability by simulation research (Schultzberg \& Muthén, 2018). Research-designedly, each participant had 28 data points, but excluding the number of omissions, only 3,368 data points were left, and the effective response rate was $99.1 \%$.

The current data included a two-level structure: repeated daily assessment data (within-person level) nested in a single individual (betweenperson level). The meaning of the results of the two-level data is different. Variability has received more attention, especially within-person variability, which inferred the individual's relevant theories of emotional variables. The between-person differences provided reasonable adjustments for the internal function of the unique insights (Brose et al., 2015). At the within-level, they were calculating the relatively stable individual differences in correlation, autoregressive relationships, and cross-lagged relationships (Hamaker \& Wichers, 2017). According to Schurman et al. (2016), autoregressive and cross-lagged parameters should be standardized in comparison. The cross-lagged parameter reflected the predictive relationship and represents a causal mechanism. However, making causal inferences needs to be aware that variables may affect the two outcome variables simultaneously to change the specific relationship (Hamaker \& Wichers, 2017). It mainly detected means (fix effects), variances (random effects), and covariates at the between-level. The random effects were adopted to capture each person's deviation from the average trajectory (Hamaker \& Wichers, 2017). If 95\% Cl did not does not contain 0, which mean that the path was significant.

In the first step, we calculated the bivariate relationship between OC symptoms and DER. The second step was to extend the model to predict SP by calculating OC symptoms, DER, and their bivariate relationships. Finally, three baseline variables (health anxiety, depression, anxiety) were controlled as covariates. The Mplus codes are presented in the supplementary materials.

\section{Results}

\subsection{Descriptive statistics}

The variables with daily measures in this study did not change over time and were relatively stable within the individuals. The results are shown in Table 1.

Table 1

Descriptive statistics $(n=122)$

\begin{tabular}{|c|c|c|c|}
\hline Scales & Range & Means & $S D$ \\
\hline $1 . \mathrm{OCl}-\mathrm{R}$ & $4-20$ & 11.61 & 3.62 \\
\hline 2.DERS & $6-28$ & 10.35 & 3.81 \\
\hline 3.ISI & $3-14$ & 5.84 & 2.56 \\
\hline 4.SHAI & $19-49$ & 29.94 & 7.31 \\
\hline 5.SAS & $20-59$ & 31.41 & 7.63 \\
\hline 6.PROMIS-Depression & $8-40$ & 15.18 & 6.43 \\
\hline
\end{tabular}

\subsection{Path correlation coefficients}


The standardized regression correlations differed between the levels. At the within-level, the correlation between OC and DEP was .03 with $95 \% \mathrm{Cl}[.00, .07]$. At the between-level, after controlling for the covariates, the correlations between DER and P1 was -.37 with $95 \% \mathrm{Cl}$ $[-.56,-.13]$; the correlations between $\mathrm{P} 1$ and $\mathrm{P} 4$ was -.26 with $95 \% \mathrm{Cl}[-.46,-.04]$. The rest of the correlations showed no significance, which ranged from -.11 to .22

\subsection{Within-person level model estimates}

\subsubsection{Autoregression effects}

Autoregressive parameters, also known as inertia, carryover, indicating how quickly an individual can regain balance after being disturbed (Hamaker \& Wichers, 2017). The standardized results showed that $\mathrm{P} 1\left(\mathrm{OC}_{\mathrm{t}-1} \rightarrow \mathrm{OC}\right)$, i.e., the autoregressive value of $\mathrm{OC}$, was positive $(\beta=.48$, $95 \% \mathrm{Cl}[.44, .53])$. It demonstrated that with severer OC symptoms in a period (12-hour), it is more difficult for individuals to restore to their original level during the next period. The influence of $\mathrm{P} 2\left(\mathrm{DER}_{\mathrm{t}-1} \rightarrow \mathrm{DER}\right)$, i.e., the autoregressive value of $\mathrm{DER}$, was also positive $(\beta=.35$, $95 \% \mathrm{Cl}[.30,40])$, which showed high inertia of DER in the last time, and it would take a longer time to return to the baseline level in the following period. The autoregressive prediction effect of OC symptoms was shown to be significantly stronger than that of DER (.48 v.s. .35).

\subsubsection{Cross-Lagged Effects}

The cross-lagged parameters represent the cascade effect of functioning or behavior cross-domain and essentially reflect the predictive relationships (Hamaker \& Wichers, 2017). On average, as shown in Fig. 1, P3 $\left(\mathrm{OC}_{\mathrm{t}-1} \rightarrow \mathrm{DER}\right)$, i.e., the cross-lagged parameter of OC affecting $\mathrm{DER}$, was shown to be statistically significant and positive $(\beta=.07,95 \% \mathrm{Cl}[.02,12])$. And $\mathrm{P} 4\left(\mathrm{DER}_{\mathrm{t}-1} \rightarrow \mathrm{OC}\right)$, i.e., the cross-lagged parameter of DER affecting OC, was also significant $(\beta=.09,95 \% \mathrm{Cl}[.05,13])$. Hence, it explained to a certain extent that both OC symptoms and DER would predict each other during the COVID-19 pandemic.

\subsection{Between-person level model estimates}

\subsubsection{Sleep Problems}

According to data analyses section, Table 2 and Fig. 2 show the results corresponding to the second step. Both OC and DER predicted SP positively, while P1 negatively predicted SP. To be specific, the slope of OC to SP was 1.07 with the $95 \% \mathrm{Cl}[.05,1.97]$; DER affecting SP was 1.20 with the $95 \% \mathrm{Cl}[.24,2.20] ; \mathrm{P} 1\left(\mathrm{OC}_{\mathrm{t}-1} \rightarrow \mathrm{OC}\right)$ negatively predicting SP was -4.25 , with $95 \% \mathrm{Cl}[-7.43,-.95]$.

\subsubsection{Covariates}

Health anxiety, anxiety, and depression were collected at baseline measurement and were viewed as time-invariant variables. As viewed from Fig. 2, HA as a significant predictor of OC symptoms $(\beta=.24,95 \% \mathrm{Cl}[.15, .34])$; anxiety positively predicted $\mathrm{DER}(\beta=.28,95 \% \mathrm{Cl}[.19, .36])$. For every unit increase in $\mathrm{HA}$, the level of OC symptoms would increase by 24 point, while for every unit increase in anxiety, the average level of people's DER would increase by .28 point. Moreover, the means (fix effects) in all steps, only P1, P2, and P3 were significant, and the variances (random effects) of all variables were significant. The variances of OC and DER decreased after controlling for covariates. 
Table 2

Parameter estimates and 95\% confidence interval of the between-level $(n=122)$

\begin{tabular}{|c|c|c|c|c|c|c|}
\hline Parameter & OC & DER & $\mathrm{P} 1\left(\mathrm{OC}_{\mathrm{t}-1} \rightarrow \mathrm{OC}\right)$ & $\mathrm{P} 2\left(\mathrm{DER}_{\mathrm{t}-1} \rightarrow \mathrm{DER}\right)$ & $\mathrm{P} 3\left(\mathrm{OC}_{\mathrm{t}-1} \rightarrow \mathrm{DER}\right)$ & $\mathrm{P} 4\left(\mathrm{DER}_{\mathrm{t}-1} \rightarrow \mathrm{OC}\right)$ \\
\hline \multicolumn{7}{|l|}{ Step1 } \\
\hline Means & $.05[-.64, .72]$ & $.01[-.70, .70]$ & $.51[.43, .61]^{\star}$ & $.35[.28, .43]^{\star}$ & $.09[.00, .17]$ & $.09[.02, .17]$ \\
\hline Variances & $10.79[7.69,15.27]^{\star}$ & $12.63[9.16,17.87]^{\star}$ & $.13[.09, .18]^{\star}$ & $.07[.04, .11]^{\star}$ & $.10[.05, .18]^{\star}$ & $.12[.08, .18]^{\star}$ \\
\hline \multicolumn{7}{|l|}{ Step 2} \\
\hline Means & $-.02[-.59, .55]$ & $-.04[-.51, .44]$ & $.51[.42, .59]^{\star}$ & $.35[.28, .43]^{\star}$ & $.08[-.01, .17]$ & $.09[.02, .17]$ \\
\hline $\begin{array}{l}\text { Residual } \\
\text { Variances }\end{array}$ & $6.73[4.78,9.44]^{\star}$ & $5.40[3.98,7.54]^{\star}$ & $.12[.09, .17]^{\star}$ & $.07[.05, .10]^{\star}$ & $.10[.06, .16]^{\star}$ & $.11[.07, .16]^{\star}$ \\
\hline $\begin{array}{l}\text { Sleep } \\
\text { Problems }\end{array}$ & $1.07[.05,1.97]^{\star}$ & $1.20[.24,2.20]^{\star}$ & $-4.25[-7.43,-.95]^{\star}$ & $-1.30[-5.08,2.59]$ & $-.48[-4.54,3.62]$ & $-.48[-4.28,3.36]$ \\
\hline $\begin{array}{l}\text { Cov.(Health } \\
\text { Anxiety) }\end{array}$ & $.24[.15, .34]^{\star}$ & $-.04[-.11, .04]$ & $.01[-.01, .02]$ & $.01[-.01, .02]$ & $-.01[-.02, .01]$ & $.01[-.01, .02]$ \\
\hline $\begin{array}{l}\text { Cov. } \\
\text { (Depression) }\end{array}$ & $.01[-.10, .12]$ & $.09[-.01, .19]$ & $-.01[-.02, .01]$ & $.01[-.00, .02]$ & $-.01[-.02, .01]$ & $.00[-.01, .02]$ \\
\hline $\begin{array}{l}\text { Cov. } \\
\text { (Anxiety) }\end{array}$ & $-.04[-.14, .06]$ & $.28[.19, .36]^{\star}$ & $-.01[-.02, .01]$ & $-.01[-.02, .01]$ & $.01[-.01, .02]$ & $-.01[-.02, .01]$ \\
\hline
\end{tabular}

Note. Results are presented as unstandardized coefficients.

\section{Discussion And Conclusions}

In our ambulatory assessment study, a two-level DSEM was used to check the bidirectional relationship between OC and DER. First, the cross-lagged effects was tested in the OC symptoms and DER (step1). Next, covariates (step2) were controlled to examine whether this relationship would change or still retain. In addition, the predictive effect of OC symptoms, DER, and their relationship, separately, on daily sleep problems was analyzed as well.

Results from the present study showed that both OC and DER had a significant autoregressive effect. After the appearance of OC and DER, they needed to take a long time to recover to the baseline level in the next period (12-hours), and OC was slower than DER to return to baseline. And it indicated that individuals tend to repeatedly choose a period of high OC and high DER or low OC and low DER. It proves that $\mathrm{OC}$ and DER have a long-term negative impact in the vicious circle.

The most important finding was that both OC and DER could predict each other. Results showed that, at within-level, OC symptoms and DER were positively related. Moreover, the cross-lagged parameters of OC symptoms and DER were significant. Specifically, by contrast, DER was shown to be a larger predictor of OC symptoms. Similar findings have been found in previous cross-sectional studies. For instance, all dimensions of DER separately had a significant correlation with OC (Yap et al., 2018; Fergus \& Bardeen, 2014). Moreover, negative emotional reactions and poor adaptation to emotions were significantly related to the improvement of OC behaviors (Coleman et al., 2011). With the higher the tendency of emotional regulation difficulties, the more difficult it was for individuals to recognize, accept and understand their own emotions (Gratz \& Roemer, 2004). In view of Gross (1998; 2013), taking OC behaviors is a means of suppressing or avoiding emotions (Gross,1998; Gross, 2013). Therefore, when perceiving their anxiety, individuals are more likely to rely on repetitive and safe behaviors to get rid of emotional distress, i.e., individuals would excessively adopt OC to cope with the pandemic. In general, the vicious circle increased the DER level in the long run and increased the impact of DER on OC.

Results also showed that, as a COVID-19-related maladaptive emotion regulation strategy, OC symptoms could positively predict and maintain DER in a period. And this cross-lagged effects was little small than the influence of DER on OC. It is consistent with the cognitive model of OC disorders (Calkins et al., 2013). Combining the pandemic situation, exaggerating that getting infections may be dysfunctional, which would push individuals to rely on social norms. Moreover, they adopted the excessive and repetitive implementation of social norms, which would be strengthened to compulsions. These compulsions may include over-checking the wearing of masks, over-focusing on epidemic information. Intrusive and threatening thoughts that continue to break in are accompanied with strong negative emotions, especially anxiety, which could cause emotional pain (Calkins et al., 2013; Stern et al., 2014). As a maladaptive emotion regulation strategy, compulsions only could temporarily relieve the emotional distress (Calkins et al., 2013). However, frequent use of compulsions would accumulate affective distress, making individuals lose confidence in their ability to regulate their emotions, thereby deepening the tendency 
to avoid emotions (Fergus \& Bardeen, 2014). When intrusive thoughts reappeared due to the lack of adaptive coping strategies, the vicious circle can only be repeated continuously. Persist in doing so, this vicious circle might bring destructive damage to individual's mental health.

In addition, the variances (random effect) of both OC and DER were decreased but still significant after controlling the covariates at the between-level. Specially, anxious emotion (including health anxiety and anxiety) showed a significant influence. In the COVID-19 pandemic, the higher the level of health anxiety, the higher the OC was, which was in line with the theory indicating that excessive worry about their health is a distorted cognition and will produce intrusive thoughts illness (Salkovskis \& Warwick, 1986). In the present study, albeit as a covariate, depression was not shown to be a significant effect, which was not consistent with findings from Yap et al. (2018). A possible explanation was that, during the early COVID-19 epidemic, anxious emotion dominated other than depression due to the uncertainty towards future (Yu et al., 2020).

Thirdly, longitudinally, maintaining a higher level of OC and DER would trigger severer sleep problems. Combining with findings from Harvey (2000) and Riemann et al. (2010), they explained that, as the obsessions and compulsions brought about by intrusive thoughts before falling asleep, the increase in negative emotions, and the decrease in positive emotions could affect the sleep process. In addition, it's worth to note that the continuous maintenance of $\mathrm{OC}$ would relieve sleep problems. A possible fact was that, with the continuous appearance of OC symptoms (i.e., washing, checking, obsessing, and mental neutralizing) could instead assure individuals when facing with potential threatening stimuli during a relative short period (e.g., 12 hours). This assurance would further lessen sleep problems.

The present research supports the importance of identifying and intervening in OC symptoms and DER and their negative impacts on sleep problems. Specifically, the public should be wary that the repeated execution of behaviors in response to the epidemic is only a temporary emotional regulation. It is necessary to identify distorted beliefs and strengthen a correct understanding of the influence of the epidemic. Cisler and Olatunji (2012) pointed out that improving the ability to regulate emotions could affect the negative emotional state caused by intrusive thoughts and improve the OC. Moreover, reasonable and adaptive emotional regulation strategies to guide were considered as an effective response to eliminating the vicious cycle of OC symptoms (Fergus \& Bardeen, 2014). Simultaneously, adaptive emotional strategies can effectively relieve insomnia (e.g., Ong \& Sholtes, 2010). Cognitive-behavioral therapy (CBT) has proven to be effective in targeting OC and DER (e.g., Clark, 2004; Berking et al., 2008). It carries out cognitive reconstruction and behavioral intervention and improves adaptive behaviors. Furthermore, CBT intends to encourage the public to perceive and accept their own emotions and to learn more adaptive emotion regulation strategies, reducing the vicious cycles.

There are some limitations that should be mentioned. First of all, most of our measurement tools have been adapted based on the epidemic situation. Even if the reliability was acceptable and good, it weakened the extent of extension to other measurement occasions. However, in the early stage of the epidemic, there was no epidemic-specific scales developed to promptly record the dynamic changes of individuals' mental state. Therefore, the advantages of such adaptation still outweighed the disadvantages. Second, the most of participants were college students, lacking special groups, such as OC patients or medical staff. Then, we did not analyze the specific-person patterns, which may be able to find specific coping patterns and obtain valuable experience at within-person level. Further research can use reliable tools, different sample groups, sample numbers, and time intervals to capture more residuals to support the reliability.

In conclusion, despite above-mentioned limitations, the present study was still the first to utilize DSEM to assess the bidirectional relationship between $\mathrm{OC}$ and DER, and their association with sleep problems.

\section{Abbreviations}

COVID-19: Coronavirus Disease of 2019

OC: Obsessive-Compulsive

DER: Difficulties in Emotion Regulation

SP: Sleep Problems

AA: Ambulatory Assessment

DSEM: Dynamic Structural Equation Modeling

ILD: Intensive Longitudinal Data

MCMC: Markov Chain Monte Carlo 
OCI-R: Obsessive-Compulsive Inventory-Revised

ICC: Intraclass Correlation

DERS: Difficulties in Emotion Regulation Scale

ISI: Insomnia Severity Index

SHAl: Short Health Anxiety Inventory

SAS: Self-Rating Anxiety Scale

\section{Declarations}

\section{Ethics approval and consent to participate}

This study has been approved by Ethics Committee of Department of Psychology, Sun Yat-Sen University (No. 2020-0712-0145) and all participants provided their informed consent online to participate in this study. In addition, all methods were carried out in accordance with relevant guidelines and regulations.

\section{Consent for publication}

All authors agree to make this paper published in BMC psychology as soon as it's accepted for publication. As for the identifying information/image(s) which include informed consent from all participants, this is not applicable.

\section{Availability of data and materials}

Materials described in the manuscript will be freely available to any researcher wishing to use them for non-commercial purposes when requesting for first or corresponding author.

\section{Competing interests}

None.

\section{Funding}

The author(s) received no specific funding for this work

\section{Authors' contributions}

Hong, D.P., conducted the statistical analysis and wrote the manuscript. Yu, M., designed the study and critically reviewed and revised the manuscript. Zhu, Y.W., Chen, R.T., Xiao, B.H., and Huang, Y.Y., participated in the study design and data collection. All authors provided critiques, and All authors contributed to and have approved the final manuscript.

\section{Acknowledgements}

None.

\section{References}

1. Alfano, C. A., \& Kim, K. L. (2011). Objective sleep patterns and severity of symptoms in pediatric obsessive compulsive disorder: a pilot investigation. Journal of anxiety disorders, 25(6), 835-839. https://doi.org/10.1016/j.janxdis.2011.04.004 
2. Altena, E., Baglioni, C., Espie, C. A., Ellis, J., Gavriloff, D., Holzinger, B., ... Riemann, D. (2020). Dealing with sleep problems during home confinement due to the COVID-19 outbreak: Practical recommendations from a task force of the European CBT-I Academy. Journal of Sleep Research, 29(4), e13052. https://doi.org/10.1111/jsr.13052

3. American Psychiatric Association. (2013). Diagnostic and statistical manual of mental health disorders: DSM-5. American Psychiatric Pub.

4. American Psychiatric Association (2014). Online assessment measures. Retrieved April 1, 2021, from http://www.psychiatry.org/practice/dsm/dsm5/online-assessment-measures

5. Amtmann, D., Kim, J., Chung, H., Bamer, A. M., Askew, R. L., Wu, S., ... Johnson, K. L. (2014). Comparing CESD-10, PHQ-9, and PROMIS depression instruments in individuals with multiple sclerosis. Rehabilitation psychology, 59(2), 220. https://doi.org/10.1037/a0035919

6. Asmundson, G. J., \& Taylor, S. (2020). How health anxiety influences responses to viral outbreaks like COVID-19: What all decisionmakers, health authorities, and health care professionals need to know. Journal of anxiety disorders, 71, 102211. https://doi.org/10.1016/j.janxdis.2020.102211

7. Asparouhov, T., Hamaker, E. L., \& Muthén, B. (2018). Dynamic structural equation models. Structural Equation Modeling: A Multidisciplinary Journal, 25(3), 359-388. https://doi.org/10.1080/10705511.2017.1406803

8. Berking, M., Wupperman, P., Reichardt, A., Pejic, T., Dippel, A., \& Znoj, H. (2008). Emotion-regulation skills as a treatment target in psychotherapy. Behavior Research and Therapy, 46(11), 1230-1237. https://doi.org/10.1016/j.brat.2008.08.005

9. Brose, A., Voelkle, M. C., Lövdén, M., Lindenberger, U., \& Schmiedek, F. (2015). Differences in the Between-Person and Within-Person Structures of Affect Are A Matter of Degree. European Journal of Personality, 29(1), 55-71. https://doi.org/10.1002/per.1961

10. Calkins, A. W., Berman, N. C., \& Wilhelm, S. (2013). Recent advances in research on cognition and emotion in OCD: a review. Current psychiatry reports, 15(5), 357. https://doi.org/10.1007/s11920-013-0357-4

11. Cisler, J. M., \& Olatunji, B. O. (2012). Emotion regulation and anxiety disorders. Current psychiatry reports, 14(3), $182-187$. https://doi.org/10.1007/s11920-012-0262-2

12. Clark, D. A. (2004). Cognitive-behavioral therapy for OCD. Guilford Press.

13. Coleman, S. L., Pieterefesa, A. S., Holaway, R. M., Coles, M. E., \& Heimberg, R. G. (2011). Content and correlates of checking related to symptoms of obsessive compulsive disorder and generalized anxiety disorder. Journal of anxiety disorders, 25(2), $293-301$. https://doi.org/10.1016/j.janxdis.2010.09.014

14. Duan, Q. Q, \& Sheng, L. (2012). Differential validity of SAS and SDS among psychiatric non-psychotic outpatients and their partners. Chinese Mental Health Journal. 26(9), 676-679. https://doi.org/10.3969/j.issn.1000-6729.2012.09.007

15. Eichholz, A., Schwartz, C., Meule, A., Heese, J., Neumüller, J., \& Voderholzer, U. (2020). Self-compassion and emotion regulation difficulties in obsessive-compulsive disorder. Clinical psychology \& psychotherapy, 27(5), 630-639. https://doi.org/10.1002/cpp.2451

16. Fergus, T. A., \& Bardeen, J. R. (2014). Emotion regulation and obsessive-compulsive symptoms: A further examination of associations. Journal of Obsessive-Compulsive and Related Disorders, 3(3), 243-248. https://doi.org/10.1016/j.jocrd.2014.06.001

17. Foa, E. B., Huppert, J. D., Leiberg, S., Langner, R., Kichic, R., Hajcak, G., \& Salkovskis, P. M. (2002). The Obsessive-Compulsive Inventory: development and validation of a short version. Psychological assessment, 14(4), 485. https://doi.org/10.1037/1040-3590.10.3.206

18. Gelman, A., Carlin, J. B., Stern, H. S., Dunson, D. B., Vehtari, A., \& Rubin, D. B. (2013). Bayesian data analysis. CRC press.

19. Gratz, K. L., \& Roemer, L. (2004). Multidimensional Assessment of Emotion Regulation and Dysregulation: Development, Factor Structure, and Initial Validation of the Difficulties in Emotion Regulation Scale. Journal of Psychopathology \& Behavioral Assessment, 26(1). https:///doi.org/10.1023/B:JOBA.0000007455.08539.94.

20. Groarke, J. M., McGlinchey, E., McKenna-Plumley, P. E., Berry, E., Graham-Wisener, L., \& Armour, C. (2021). Examining temporal interactions between loneliness and depressive symptoms and the mediating role of emotion regulation difficulties among UK residents during the COVID-19 lockdown: Longitudinal results from the COVID-19 Psychological Wellbeing Study. Journal of Affective Disorders, 285, 1-9. https://doi.org/10.1016/j.jad.2021.02.033

21. Gross, J. J. (1998). The emerging field of emotion regulation: An integrative review. Review of general psychology, 2(3), $271-299$. https://doi.org/10.1037/1089-2680.2.3.271

22. Gross, J. J. (2013). Emotion regulation: taking stock and moving forward. Emotion, 13(3), 359. https://doi.org/10.1037/a0032135

23. Görgen, S. M., Hiller, W., \& Witthöft, M. (2014). Health anxiety, cognitive coping, and emotion regulation: A latent variable approach. International Journal of Behavioral Medicine, 21(2), 364-374. https:///doi.org/10.1007/s12529-013-9297-y

24. Harvey, A. G. (2000). Pre-sleep cognitive activity: A comparison of sleep-onset insomniacs and good sleepers. British Journal of Clinical Psychology, 39(3), 275-286. https://doi.org/10.1348/014466500163284

Page $10 / 13$ 
25. Hamaker, E. L., Asparouhov, T., Brose, A., Schmiedek, F., \& Muthén, B. (2018). At the frontiers of modeling intensive longitudinal data: Dynamic structural equation models for the affective measurements from the COGITO study. Multivariate Behavioral Research, 53(6), 820-841. https://doi.org/10.1080/00273171.2018.1446819

26. Hamaker E., Wichers M. (2017). No time like the present: Discovering the hidden dynamics in intensive longitudinal data. Current Directions in Psychological Science, 26, 10-15. https://doi.org/10.1177/0963721416666518

27. Hoyle, R. H. (Ed.). (2012). Handbook of structural equation modeling. Guilford press.

28. Hu, T., Zhang, D., Wang, J., Mistry, R., Ran, G., \& Wang, X. (2014). Relation between emotion regulation and mental health: a metaanalysis review. Psychological reports, 114(2), 341-362. https://doi.org/10.2466/03.20.PR0.114k22w4

29. Jahrami, H., BaHammam, A. S., Bragazzi, N. L., Saif, Z., Faris, M., \& Vitiello, M. V. (2021). Sleep problems during the COVID-19 pandemic by population: a systematic review and meta-analysis. Journal of Clinical Sleep Medicine, 17(2), $299-313$. https://doi.org/10.5664/jcsm.8930

30. Li, J., Han, Z. R., Gao, M. M., Sun, X., \& Ahemaitijiang, N. (2018). Psychometric properties of the Chinese version of the Difficulties in Emotion Regulation Scale (DERS): Factor structure, reliability, and validity. Psychological Assessment, 30(5), e1-e9. https://doi.org/10.1037/pas0000582

31. McNeish, D., \& Hamaker, E. L. (2020). A primer on two-level dynamic structural equation models for intensive longitudinal data in Mplus. Psychological methods, 25(5), 610. https://doi.org/10.1037/met0000250

32. Morin, C. M. (1993). Insomnia: Psychological assessment and management. Guilford press.

33. Moritz, S., Fink, J., Miegel, F., Nitsche, K., Kraft, V., Tonn, P., \& Jelinek, L. (2018). Obsessive-compulsive disorder is characterized by a lack of adaptive coping rather than an excess of maladaptive coping. Cognitive Therapy and Research, 42(5), 650-660. https://doi.org/10.1007/s10608-018-9902-0

34. Muthen, B. O. (2019). How to get the ICC in DSEM. https://www.statmodel.com/download/ICCinDSEM.pdf

35. Olatunji, B. O., Ferreira-Garcia, R., Caseras, X., Fullana, M. A., Wooderson, S., Speckens, A., ... Mataix-Cols, D. (2014). Predicting response to cognitive behavioral therapy in contamination-based obsessive-compulsive disorder from functional magnetic resonance imaging. Psychological medicine, 44(10), 2125. https://doi.org/10.1017/S0033291713002766

36. Ong, J., \& Sholtes, D. (2010). A mindfulness-based approach to the treatment of insomnia. Journal of clinical psychology, 66(11), 1175-1184. https://doi.org/10.1002/jclp.20736

37. Riemann, D., Spiegelhalder, K., Feige, B., Voderholzer, U., Berger, M., Perlis, M., \& Nissen, C. (2010). The hyperarousal model of insomnia: a review of the concept and its evidence. Sleep medicine reviews, 14(1), 19-31. https://doi.org/10.1016/j.smrv.2009.04.002

38. Salkovskis, P.M. \& Warwick, H.M.(1986). Morbid preoccupations, health anxiety and reassurance: a cognitive-behavioural approach to hypochondriasis. Behaviour Research and Therapy, 24(5), 597-602. https://doi.org/10.1016/0005-7967(86)90041-0.

39. Salkovskis, P. M., Rimes, K. A., \& Warwick, H. M. C. (2002). The Health Anxiety Inventory: development and validation of scales for the measurement of health anxiety and hypochondriasis. Psychological medicine, 32(5), 843.

https://doi.org/10.1017\S0033291702005822

40. Schultzberg, M., \& Muthén, B. (2018). Number of subjects and time points needed for multilevel time-series analysis: A simulation study of dynamic structural equation modeling. Structural Equation Modeling: A Multidisciplinary Journal, 25(4), 495-515. https://doi.org/10.1080/10705511.2017.1392862

41. Schuurman N. K., Ferrer E., de Boer-Sonnenschein M., Hamaker E. L. (2016). How to compare cross-lagged associations in a multilevel autoregressive model. Psychological Methods, 21, 206-21. https://doi.org/10.1037/met0000062

42. Sunderland, M., Newby, J. M., \& Andrews, G. (2013). Health anxiety in Australia: prevalence, comorbidity, disability and service use. The British Journal of Psychiatry, 202(1), 56-61. https://doi.org/10.1192/bjp.bp.111.103960

43. Stern, M. R., Nota, J. A., Heimberg, R. G., Holaway, R. M., \& Coles, M. E. (2014). An initial examination of emotion regulation and obsessive compulsive symptoms. Journal of Obsessive-Compulsive and Related Disorders, 3(2), $109-114$. https://doi.org/10.1016/j.jocrd.2014.02.005

44. Tang, S.Q., Wang, J.P., Tang, T., \& Zhao, L.N. (2011). Reliability and Validity of Obsessive-compulsive Inventory-revised in Chinese College Students. Chinese Journal of Clinical Psychology, 19(5), 619-621. https://doi.org/10.16128/j.cnki.1005-3611.2011.05.038.

45. Tavernier, R., \& Willoughby, T. (2015). A longitudinal examination of the bidirectional association between sleep problems and social ties at university: The mediating role of emotion regulation. Journal of youth and adolescence, 44(2), 317-330. https://doi.org/10.1007/s10964-014-0107-x

46. Timpano, K. R., Carbonella, J. Y., Bernert, R. A., \& Schmidt, N. B. (2014). Obsessive compulsive symptoms and sleep difficulties: Exploring the unique relationship between insomnia and obsessions. Journal of psychiatric research, 57, 101-107. 
https://doi.org/10.1016/j.jpsychires.2014.06.021

47. Trull, T. J., \& Ebner-Priemer, U. (2013). Ambulatory assessment. Annual review of clinical psychology, 9, 151-176. https://doi.org/10.1146/annurev-clinpsy-050212-185510

48. Tsypes, A., Aldao, A., \& Mennin, D. S. (2013). Emotion dysregulation and sleep difficulties in generalized anxiety disorder. Journal of anxiety disorders, 27(2), 197-203. https://doi.org/10.1016/j.janxdis.2013.01.008

49. Yap, K., Mogan, C., Moriarty, A., Dowling, N., Blair-West, S., Gelgec, C., \& Moulding, R. (2018). Emotion regulation difficulties in obsessivecompulsive disorder. Journal of clinical psychology, 74(4), 695-709. https://doi.org/10.1002/jclp.22553

50. Yu, L., Buysse, D. J., Germain, A., Moul, D. E., Stover, A., Dodds, N. E., ... Pilkonis, P. A. (2012). Development of short forms from the $\mathrm{PROMIS}^{\mathrm{TM}}$ sleep disturbance and sleep-related impairment item banks. Behavioral sleep medicine, 10(1), 6-24.

https://doi.org/10.1080/15402002.2012.636266

51. Yu, M., Huang, Y.Y., Chen,R.t., Xiao, B.H, Wang, J.P., Zhu, Y.W (2020). Investigation of cognitive, emotional and behavioral responses among different populations during the COVID-19 epidemic. SiChuan Metal Health, 33(3), 197-201. https://doi.org/10.11886/scjsws20200228001

52. Zhang, Y.Q., Liu, R., Li, G., Mao, S., \& Yuan, Y. (2015). The reliability and validity of a Chinese-version Short Health Anxiety Inventory: An investigation of university students. Neuropsychiatric Disease and Treatment, 11,1739. https://doi.org/10.2147/NDT.S83501.

53. Zung, W. W. (1971). A rating instrument for anxiety disorders. Psychosomatics: Journal of Consultation and Liaison Psychiatry. https://doi.org/10.1016/S0033-3182(71)71479-0

\section{Figures}

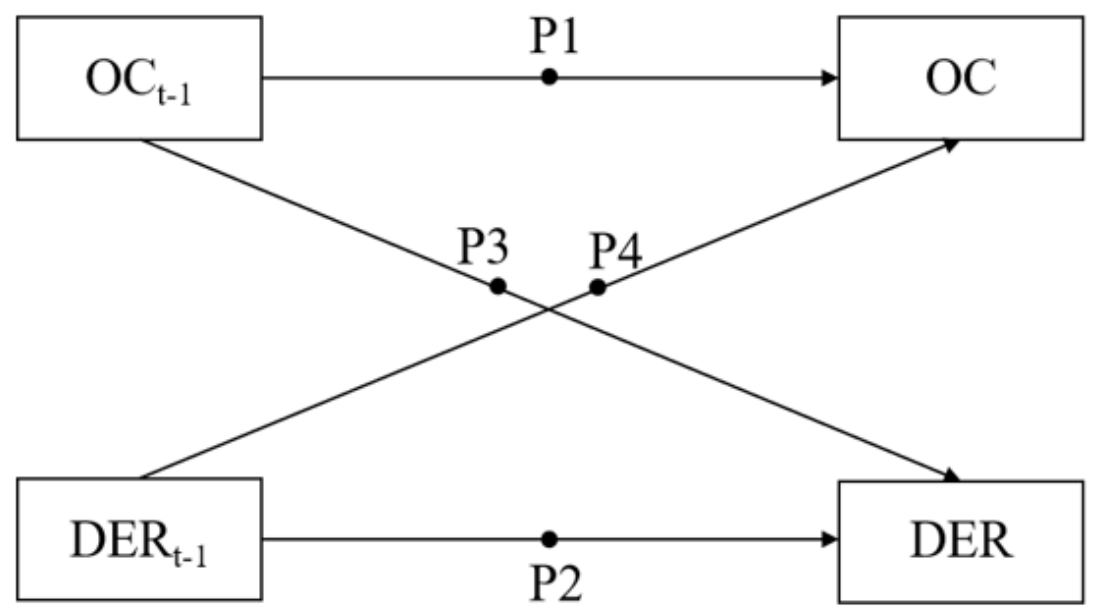

\section{Figure 1}

Autoregressive and cross-lagged parameter paths of the within-level. Note. OC is obsessive-compulsive symptoms, and DER is difficulties in emotion regulation. $\mathrm{P} 1$ and $\mathrm{P} 2$ represent the autoregressive path of $\mathrm{OC}$ and DER, respectively, while P3 and P4 represent the cross-lagged paths. After controlling for the covariates, the effects were still significant. 


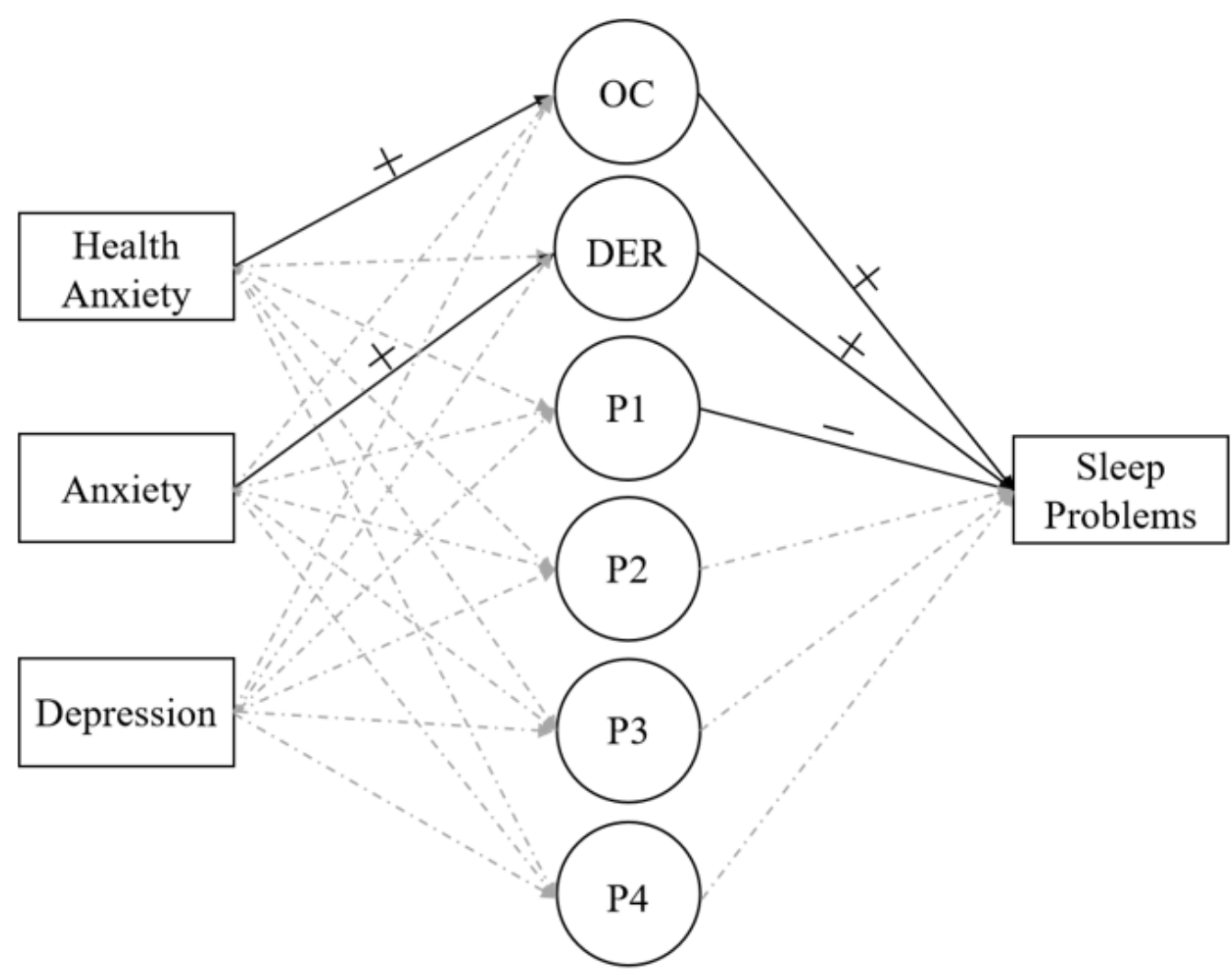

Figure 2

The between-level of Step 2. Note. OC is Obsessive-Compulsive Symptoms, while DER is Difficulties in Emotion Regulation. P1, P2, P3, P4 are the autoregressive and cross-lagged parameters of $\mathrm{OC}$ and DER, respectively. At the between-level, time-invariant covariates were controlled for OC, DER, P1, P2, P3, and P4.

\section{Supplementary Files}

This is a list of supplementary files associated with this preprint. Click to download.

- SupplementaryMaterials.docx 